\title{
Summation and transfer of negative occasion setting
}

\author{
JAVIER R. MORELL and PETER C. HOLLAND \\ Duke University, Durham, North Carolina
}

\begin{abstract}
Two experiments examined the summation and transfer of negative occasion setting in serial feature negative $(X \rightarrow A-/ A+)$ and negative patterning discriminations $(X \rightarrow A-/ X+/ A+)$ in a discrete-trial operant task with rats. With both discrimination procedures, negative occasion setting transferred readily (but not perfectly) to cues trained as targets in another similar discrimination procedure, but not to cues that were separately trained. Furthermore, with both discrimination procedures, the negative occasion-setting powers of features from two individual discriminations summed, both when those features were trained with the same targets and when they were trained with different targets. After negative patterning discriminations, in which the feature $(\mathrm{X})$ cues were separately reinforced, this summation of negative occasion setting occurred despite the concomitant summation of the features' excitatory control in the absence of an explicit target cue. These data, which replicated and extended previously reported data from Pavlovian feature negative procedures, are discussed in the context of hierarchical, generalization, and network models of discrimination learning.
\end{abstract}

Holland (1985) distinguished between simple Pavlovian conditioned inhibition and a modulatory function he termed negative occasion setting. This distinction grew from work with various Pavlovian A+/XA- feature negative discrimination procedures, in which a stimulus, the target (A), is reinforced when it is presented alone, but nonreinforced when it is accompanied by another cue, the feature $(\mathrm{X})$. Although, with all these procedures, rats learned to respond on $\mathrm{A}+$ trials but not on XA-trials, several aspects of the data suggested that $X$ acquired different powers depending on the temporal arrangement of the $\mathrm{X}$ and $\mathrm{A}$ elements on compound trials. Specifically, Holland (1985) claimed that when X and A were presented simultaneously in training, $X$ acquired a direct inhibitory association with a representation of the unconditioned stimulus (US), but when $X$ preceded $A, X$ acquired the ability to modulate the effectiveness of the association between $\mathrm{A}$ and the US (negative occasion setting).

Two distinctions between the consequences of serial and simultaneous presentation of the elements on compound training trials are the effects of counterconditioning of the feature and the breadth of transfer of the feature's powers. First, $\mathrm{X}$-US counterconditioning pairings after training with serial $X \rightarrow A$ compounds had relatively little effect on X's ability to suppress responding to $A$. But counterconditioning of $\mathrm{X}$ after training with simultaneous $\mathrm{XA}$ compounds rendered $X$ unable to inhibit responding to A. Indeed, in some cases, $X$ 's counterconditioned response tendencies summed with A's, so that the XA compound elicited more responding than did $\mathrm{A}$ alone.

This research was supported by a grant from the National Science Foundation. We thank Marie Crock and Stephanie Nevels for technical assistance. Correspondence can be directed to either author at the Department of Psychology: Experimental, Duke University, Durham, NC 27708-0086.
Second, after serial training, X's ability to suppress responding elicited by cues other than A was severely limited, whereas after simultaneous training, $X$ 's inhibitory powers were quite general. After serial training, $X$ suppressed responding controlled by cues that had been trained as targets in other $\mathrm{X} \rightarrow \mathrm{A}-/ \mathrm{A}+$ discriminations, but had litthe or no effect on responding controlled by cues trained with a variety of other procedures (Holland, 1989; Lamarre \& Holland, 1987). Conversely, after simultaneous training, $X$ suppressed responding regardless of the training history of its test target.

Recently, Holland (1991) noted a similar distinction in rats' solution of discrete-trial operant serial and simultaneous feature negative discriminations. These operant procedures were like those of his earlier Pavlovian experiments, with the exception that the food reinforcer was delivered on A+ trials only when an operant leverpress response occurred. As in the Pavlovian conditioning experiments, Holland (1991) found both that counterconditioning of the feature had greater deleterious effects after simultaneous feature negative discrimination training than after serial feature negative discrimination training and that only simultaneously trained Xs inhibited responding controlled by another, separately trained cue. The experiments reported in this article extend Holland's (1991) findings in several ways.

First, these experiments examined the importance of a target cue's training history for the transfer of a feature's negative occasion-setting power in this operant procedure. We compared transfer to a cue during which responding had been consistently reinforced with transfer to a cue that had been trained as the target in another serial feature negative discrimination, a procedure that yielded an acceptable transfer target with Pavlovian procedures (e.g., Holland, 1989). 
Second, we examined the summation of the powers of two negative occasion-setting features. Summation of the simple associative powers of conditioned stimuli (CSs) is a property basic to most theories of conditioning, but the summation of the strengths of conditional cues like occasion setters is by no means a forgone conclusion. For example, after training two independent matching-tosample discriminations, Maki and Leuin (1972) found the individual sample cues to be more effective than a compound of the two sample cues.

The summation tests also provided another way of addressing the specificity of coding of the target in negative occasion setting. We compared the summation of the strengths of two negative occasion setters originally trained with the same target excitor to summation obtained with two negative occasion setters trained with different targets. To the extent that a negative occasion setter's power is specific to its original target, combining features trained with different targets might be expected to yield less summation than would compounding of features trained with a common target.

Third, we examined these summation and transfer effects in serial negative patterning $(\mathrm{X} \rightarrow \mathrm{A}-, \mathrm{A}+, \mathrm{X}+)$, as well as serial feature negative $(X \rightarrow A-, A+)$ procedures. Because the reinforcement of $X$ in the negative patterning procedure apparently minimizes the contribution of X's simple inhibitory powers (e.g., Holland, 1989), performance in this procedure is more likely to reflect X's occasion-setting powers. Furthermore, the summation of two features' negative occasion-setting powers would be particularly compelling if, at the same time, their simple excitatory powers summed.

\section{EXPERIMENT 1}

In Experiment 1, we examined the summation and transfer of negative occasion setting in serial feature negative discrimination training procedures. Three groups of rats received training on a common $\mathrm{X} \rightarrow \mathrm{A}-\mathrm{A}+$ discrimination, but differed in the treatment given to another pair of cues, $\mathrm{Y}$ and $\mathrm{B}$. In Group One, $\mathrm{Y}$ was trained as the feature in a second $\mathrm{Y} \rightarrow \mathrm{A}-/ \mathrm{A}+$ discrimination, with the same target cue as was used in the common discrimination, and responding was reinforced during separate presentations of B. In Group Two, $Y$ and B were trained in a $\mathrm{Y} \rightarrow \mathrm{B}-/ \mathrm{B}+$ discrimination. In Group Con, $\mathrm{Y}$ was presented alone, without a target, and responding was reinforced during separate presentations of B. Finally, all groups received several test sessions, which examined the ability of $\mathrm{X}, \mathrm{Y}$, and a compound of $\mathrm{X}$ and $\mathrm{Y}$ to modulate responding in the presence of $A$ and $B$.

\section{Method}

Subjects and Apparatus. The subjects were 12 male and $12 \mathrm{fe}-$ male albino rats bred in the Psychology Department of Duke University. They were about 170 days old at the beginning of the experiment and had not been involved in previous research. The rats were maintained at $85 \%$ of their ad-lib body weights throughout the experiment by limiting their access to food. Water was available at all times in their individual home cages.

There were eight identical experimental chambers, each $22.9 \times 20.3 \times 20.3 \mathrm{~cm}$. The front and back walls of each chamber were aluminum; the side walls and top were clear acrylic. A food cup was recessed behind a $5 \times 5 \mathrm{~cm}$ opening in the front wall; the bottom of the opening was $2 \mathrm{~cm}$ from the floor, and its center was $2 \mathrm{~cm}$ to the right of the center of the front wall. A 6-W jewelled lamp (panel light) was centered on the front wall, $4 \mathrm{~cm}$ above the top of the food cup opening. Four centimeters left of the food cup opening was a $2 \times 2 \mathrm{~cm}$ lever, mounted $3 \mathrm{~cm}$ above the floor. The floor of the chamber was composed of $0.48-\mathrm{cm}$ stainless steel rods spaced $1.9 \mathrm{~cm}$ apart. Each of the chambers was enclosed in a soundattenuating shell. A speaker for delivering the auditory CSs was mounted on one inside wall of that shell even with the top of the chamber, $2 \mathrm{~cm}$ in front of and $10 \mathrm{~cm}$ to the left of the front wall of the chamber. A 6-W lamp (door light) was mounted on the opposite wall (door) of the shell, $5 \mathrm{~cm}$ above the top of the chamber and $10 \mathrm{~cm}$ to the right of the front wall of the chamber. Each shell was enclosed in another sound-attenuating box. Constant background noise $(62.5 \mathrm{~dB})$ was provided by a ventilating fan on each box.

Procedure. First, the rats were trained to press the lever. In the first, 60 -min, session, the rats received $200.3-\mathrm{ml}$ deliveries of $0.2 \mathrm{M}$ sucrose (the reinforcer used throughout these experiments) on a variable-time 2 -min schedule. Leverpresses were reinforced during that period and during the remaining $40 \mathrm{~min}$ of the session. In the second session, each rat was allowed to remain in its chamber until it had made about 50 leverpresses or $1 \mathrm{~h}$ had elapsed. Leverpresses were reinforced, but there were no response-independent sucrose presentations in this session. About half the rats needed another identical session to reach that criterion.

The next eight 64-min sessions were designed to place leverpressing under the control of the two excitors to be used in discrimination training and to eliminate responding during the intertrial interval (ITI). In the first five sessions, there were 16 randomly intermixed illuminations of the panel light (B) and 16 presentations of a 72-dB auditory stimulus (A). For half of the rats in each group, the auditory cue was a white noise; for the other half, the auditory cue was a $1500-\mathrm{Hz}$ tone. Each leverpress during presentation of A or B was reinforced with a $0.3-\mathrm{ml}$ delivery of $0.2 \mathrm{M}$ sucrose. Each cue was $30 \mathrm{sec}$ long in the first session and $15 \mathrm{sec}$ long in the second session of this phase. Each cue was gradually shortened to $5 \mathrm{sec}$ over the course of the third session, and all cue presentations were $5 \mathrm{sec}$ in duration in the fourth and fifth sessions. In the sixth session, the number of each trial type was reduced to eight; in the seventh and eighth sessions, only four of each type of trial were given.

Next, 4 male and 4 female rats were randomly assigned to each of three groups, and discrimination training was started. In each of the 4064 -min discrimination training sessions, all rats received four kinds of trials, randomly intermixed, with intertrial intervals ranging from 4 to $12 \mathrm{~min}(M=8 \mathrm{~min})$. The subjects in all three groups received identical training with the primary discrimination: $\mathrm{A}+$ trials, in which each leverpress during the 5-sec A presentation was reinforced, and $X \rightarrow A-$ trials, which comprised a 5-sec illumination of the door light $(X)$, followed, after a 5-sec empty trace interval, by a 5 -sec presentation of $A$, during which reinforcement was not available. Similarly, the rats in all three groups received $\mathrm{B}+$ trials, in which each leverpress during the 5-sec B presentation was reinforced. In addition, the rats in Group One received $\mathrm{Y} \rightarrow \mathrm{A}-$ trials, in which a 5-sec presentation of a 68-dB, 8-Hz clicker (Y) was followed, after a 5-sec trace interval, by a 5-sec presentation of $A$. The rats in Group Two received $Y \rightarrow B-$ trials, in which the 5-sec $Y$ was followed, after a 5-sec trace interval, by a 5-sec $B$ presentation. The rats in Group Con received 5-sec Y-alone presentations. In each of the first 20 sessions, there were two of each 
of the four trial types in each session; in the last 20 sessions, there were three of each nonreinforced trial type and one of each reinforced trial type.

All subjects then received six 88-min test sessions. Sessions 1, 4 , and 6 evaluated summation and transfer with the A target; Sessions 2,3 , and 5 evaluated summation and transfer with the B target. Each session included seven types of trials. First, feature $\rightarrow$ target trials comprised a 5-sec presentation of $X, Y$, or a simultaneous $5-\sec X Y$ compound, followed (after a 5-sec trace interval) by a $5-\sec A$ or B presentation, depending on the test session. Second, target-alone trials consisted of a 5-sec presentation of the tested target in the absence of any feature. Third, feature-alone trials comprised 5-sec presentations of $\mathrm{X}, \mathrm{Y}$, or $\mathrm{XY}$ alone. Responding was recorded during those cues, as well as during the periods of time after those cues, when one of the targets would otherwise have occurred. In each session, there were two of each of the feature-target and targetonly trials, and one of each of the feature-only trials. No reinforcement was available during any of these sessions.

Data analysis. As in previous studies (e.g., Holland, 1991), we recorded the percentage of trials on which at least one response occurred, response rate, and the latency to the first response, during each 5-sec interval of the CS presentations and the 5-sec preCS interval. Also as in previous studies, response latency proved the most sensitive measure. For the sake of economy, only that index is presented in the present experiment. Distribution-free inferential statistics were used throughout, with the level of significance defined as .05 .

\section{Results and Discussion}

Feature negative discrimination learning proceeded slowly, especially with the B target. One rat in Group Two and two rats in Group Con ceased responding during reinforced B trials (and thus failed to receive the scheduled reinforcement); their data were discarded from the analyses. By the end of training, all remaining subjects responded with slower latencies during the target on nonreinforced compound trials than on reinforced target-alone trials. Discrimination performance over the final five sessions of training is shown in Table 1 . Training was halted at numerically poor levels of discrimination performance intentionally, to minimize ceiling effects during subsequent summation tests.

Figure 1 shows, combined over the six test sessions, the response latencies on compound and target-alone trials (the rats never responded on feature-alone trials). Consider first the performance of Group One (top panel), in which the $X$ and $Y$ features were both trained with the
A target. Both of the individual features and the compound (XY) feature slowed responding to the subsequent A target [Wilcoxon $T \mathrm{~s}(8) \leq 3$; see left bars]. Furthermore, the compound feature slowed responding more than did either $\mathrm{Y}$ or $\mathrm{X}$ alone $[T \mathrm{~s}(8)=0]$. Thus, the abilities of $X$ and $Y$ to inhibit responding to $A$ apparently summed. However, $X, Y$, and the $X Y$ compound did not slow responding to the separately trained $Y$ excitor $[T s(8) \geq 16]$. Thus, consistent with Holland's (1991) findings, there was no transfer of negative occasion setting to a separately trained excitor.

The center panel of Figure 1 shows the test performance of Group Two, in which the $X$ feature had been trained with the $A$ target, and the $Y$ feature had been trained with the B target. Some transfer of the features' occasionsetting power was evident, but that transfer was less than complete. Although $X$ reliably slowed responding to the B transfer target $[T(7)=0]$, Y did not significantly affect responding to the $A$ transfer target $[T(7)=11.5]$. More important, both features slowed responding to their original targets more than responding to the transfer targets: The difference between response latencies on compound trials and on target-alone trials was reliably greater for the original feature-target combination than for the transfer combination for both the $X$ feature $[T(7)=4]$ and the $Y$ feature $[T(7)=1]$.

As in Group One, the negative occasion-setting powers of the two features summed: The XY compound slowed responding to the $A$ target and the $B$ target more than did either feature alone $[T \mathrm{~s}(7) \leq 2]$. However, the summation effect was apparently smaller in Group Two than in Group One. The difference in latency to respond to $A$ between $\mathrm{X} \rightarrow \mathrm{A}$ trials (the training sequence) and compound $\mathrm{XY} \rightarrow \mathrm{A}$ trials was greater in Group One [Mann-Whitney $U(8,7)=14$ ]. That is, X's ability to suppress responding to the original $\mathrm{A}$ target was enhanced by the addition of the $Y$ feature to a greater extent in Group One than in Group Two. Unfortunately, this conclusion is weakened by the possibility of ceiling effects in Group Two. The true effects of combining $X$ and $Y$ in that group might have been masked by the already high performance level with the $\mathrm{X} \rightarrow \mathrm{A}$ discrimination.

The bottom panel of Figure 1 shows the test performance of Group Con, in which the $\mathrm{X}$ feature had been

Table 1

Response Latencies Over the Final Block of Five Discrimination Training Sessions

Experiment 1:

\begin{tabular}{|c|c|c|c|c|c|c|c|c|c|c|c|}
\hline \multicolumn{4}{|c|}{ Group One } & \multicolumn{4}{|c|}{ Group Two } & \multicolumn{4}{|c|}{ Group Con } \\
\hline $\mathbf{X} \rightarrow \mathbf{A -}$ & At & $\mathrm{Y} \rightarrow \mathrm{A}-$ & B+ & $X \rightarrow A-$ & At & $\mathrm{Y} \rightarrow \mathrm{B}-$ & $\mathbf{B +}$ & $\mathbf{X} \rightarrow \mathbf{A}-$ & A+ & $\mathrm{Y} \rightarrow \mathrm{O}-$ & B+ \\
\hline 3.57 & 1.66 & 2.73 & 2.64 & 3.73 & 1.66 & 3.45 & 3.09 & 4.12 & 1.44 & 5.00 & 3.31 \\
\hline
\end{tabular}

Experiment 2:

\begin{tabular}{|c|c|c|c|c|c|c|c|c|c|c|c|c|c|c|c|c|}
\hline \multicolumn{6}{|c|}{ Group One } & \multicolumn{6}{|c|}{ Group Two } & \multicolumn{5}{|c|}{ Group Con } \\
\hline$X \rightarrow A-$ & At & $X+$ & $\mathrm{Y} \rightarrow \mathrm{A}-$ & $\mathrm{B}+$ & $\overline{Y+}$ & $\mathrm{X} \rightarrow \mathrm{A}-$ & At & $\mathrm{X}+$ & $\mathrm{Y} \rightarrow \mathrm{B}-$ & B+ & $\overline{Y+}$ & $\bar{X} \rightarrow A-$ & A+ & $x+$ & B+ & $\overline{Y+1-}$ \\
\hline 2.09 & 1.39 & 2.25 & 2.22 & 2.62 & 1.88 & 2.19 & 1.08 & 1.93 & 2.78 & 2.06 & 1.94 & 2.52 & 1.19 & 2.37 & 2.30 & 2.86 \\
\hline
\end{tabular}

Note-Entries are in seconds. On compound trials and on feature-alone trials $(X+$ and $Y+)$ in Experiment 2, latencies refer to latency to respond during the target or empty interval. $\mathrm{Y}=$ clicker, $\mathrm{X}=$ door light, $\mathrm{A}=$ noise or tone, $\mathrm{B}=$ panel light, $+=$ reinforcement available, $-=$ no reinforcement available. 

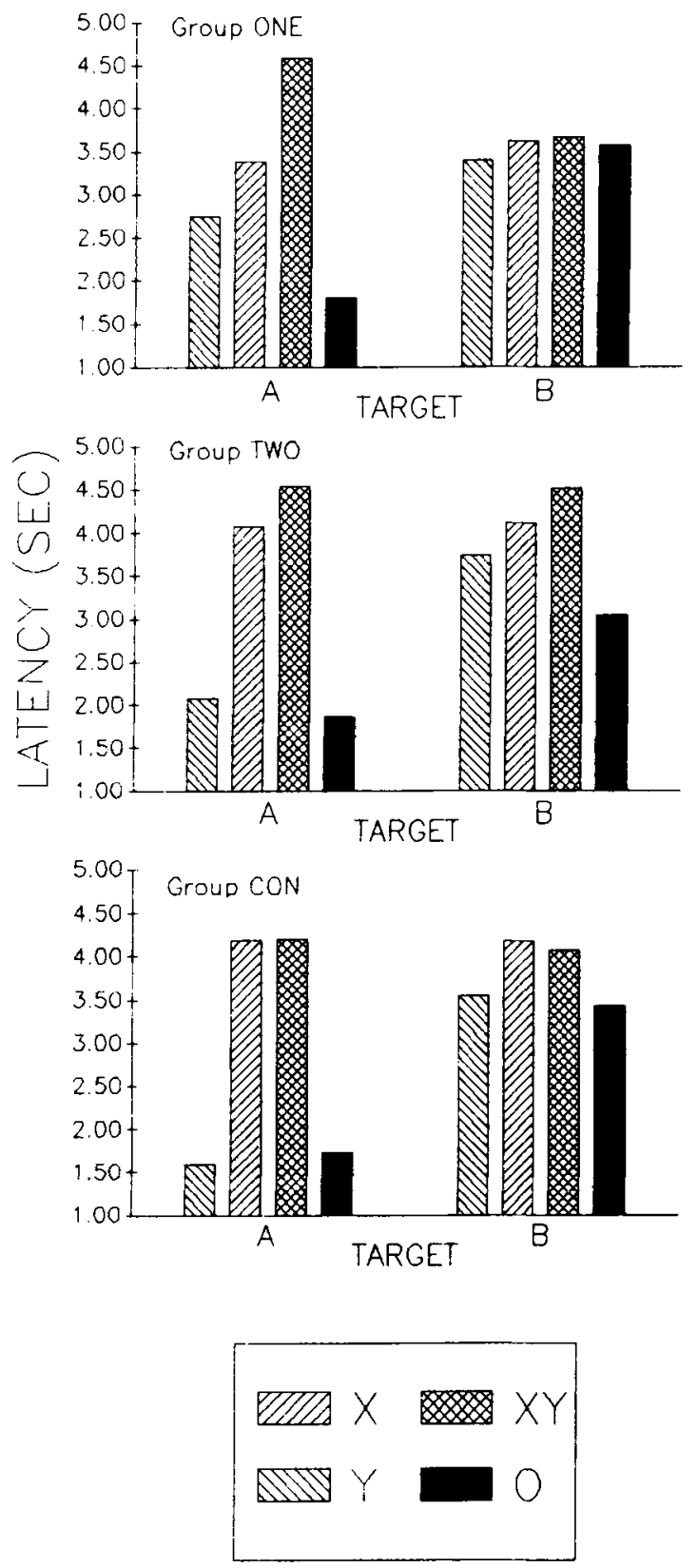

Figure 1. Test performance in Experiment 1. In each panel, the left set of bars shows responding on test trials that included the $A$ (noise/tone) target, and the right set of bars shows responding on test trials that included the B (panel light) target. Shading of the bars (see legend) signifies whether a target was preceded by the $X$ (door light), Y (clicker), XY (clicker + door light compound), or no (O) feature. There was no responding on feature-alone trials.

trained in a feature negative discrimination with the A target, but the $\mathrm{Y}$ cue was simply nonreinforced (and thus not trained as an occasion setter). Y did not slow responding to either the A target or the B target $[T \mathrm{~s}(6)=9]$ and did not enhance $X$ 's ability to slow responding to $A$ $[T(6)=8]$, suggesting that the summation observed in Groups One and Two was due to the training contingen- cies of those groups rather than, for example, the mere presence of the two features at the time of testing. The difference between responding during the $A$ target after the $X Y$ compound feature and after the $X$ feature, the measure of summation, was greater in both Group One $[U(8,6)$ $=5]$ and Group Two $[U(7,6)=11]$ than in Group Con. Also, as in Group One, the $X$ feature failed to significantly slow responding to the separately reinforced $B$ target $[T(6)=6]$.

It is worth pointing out that, in all three groups, the latencies to respond to the transfer $B$ target were longer than those to the original A target. Casual observations suggest that this difference was related to the occurrence of orienting responses to the onset of the panel light $B$ cue, which interfered with approach to the lever. Although this latency difference complicates comparisons of the magnitudes of the discriminations involving $A$ with those involving B and raises the possibility of ceiling effects, the major conclusions of Experiment 1 are unaffected. First, although some transfer to the separately trained B target in Groups One and Con might have been masked by ceiling effects, the critical transfer finding is that transfer to B was greater in Group Two than in the former groups. Note that the latency of responding to $B$ was equivalent in the three groups. Second, the reliable summation observed with both the A target and the B target in Group Two demonstrates adequate sensitivity of the latency measure in both ranges.

In summary, although the negative occasion setters' failure to suppress responding controlled by the separately reinforced B is consistent with Holland's (1983) claim that occasion setters act by modulating the effectiveness of a particular CS-US association, the transfer and summation of the occasion-setting powers of features that were trained with different targets in Group Two indicate a more general site of action. At the same time, Experiment 1 provided evidence for some specificity of occasion setters' action even among targets of occasion setters. Transfer discrimination performance was inferior to performance on the original discriminations in Group Two, and there was some evidence of less summation when the summed features were trained with different targets (Group Two) than when they were trained with a common feature (Group One).

It might be argued that the summation effects observed here reflect the summation of simple inhibitory tendencies rather than the summation of negative occasion setting. We consider this argument unlikely for two reasons. First, in our laboratory, simple conditioned inhibition is revealed only weakly in serial feature $\rightarrow$ target tests. Second, simple inhibition, unlike negative occasion setting, typically transfers to separately trained cues. Of course, it could be further argued that summation tests would especially reveal weak inhibition, and that even simple inhibition transfers less readily to separately trained cues. Experiment 2 provides additional evidence that negative occasion setting, distinct from simple inhibition, exhibits differential summation and transfer effects. 


\section{EXPERIMENT 2}

One of the hallmarks of negative occasion setting, relative to conditioned inhibition in our conditioning preparations, is its resistance to the effects of reinforcement. In Experiment 2, we examined the transfer and summation of negative occasion setting established to cues in serial negative patterning discriminations $(X \rightarrow A-/ A+/$ $X+)$, in which responding was not reinforced during the serial $\mathrm{X} \rightarrow \mathrm{A}$ compound, but was reinforced during $\mathrm{A}$ alone presentations, and on $\mathrm{X}$-alone presentations, during an empty interval that corresponded to the time when A would have been delivered on $X \rightarrow A$ trials. Because the simple associative relations of the features with the US would be excitatory, this procedure separated a feature's occasion setting and simple associative powers.

Other than the additional presentations of $\mathrm{X}$ and $\mathrm{Y}$, during which responding was reinforced, the procedures of Experiment 2 were identical to those of Experiment 1. Three groups of rats received training on an $\mathrm{X} \rightarrow \mathrm{A}-1$ $\mathrm{A}+/ \mathrm{X}+$ discrimination, but differed as to whether a second feature $(Y)$ was trained in a negative patterning discrimination with the same target (Group One), different targets (Group Two), or no target (Group Con).

\section{Method}

Subjects and Apparatus. The subjects were 13 male and 11 female albino rats, about 105 days old at the beginning of the experiment. They were maintained as in Experiment 1. The apparatus was the same as that used in Experiment 1.

Procedures. Initially, the rats were trained to press the lever in the same manner as in Experiment 1. Hand shaping was used if necessary. They were then given five 64 -min pretraining sessions designed to place leverpressing under the control of four different stimuli in all three groups: the noise/tone (A), panel light (B), door light $(\mathrm{X})$, and clicker $(\mathrm{Y})$ cues used in Experiment 1 . In the first two of these sessions, there were eight presentations of each cue, and each leverpress during each cue was reinforced. In the first session, each cue was $30 \mathrm{sec}$ long; in the second session, each was 15 sec long.

The third pretraining session was designed to bring responding under the control of 5-sec A and 5-sec B cues by reducing cue duration in steps. The first five presentations of each cue were each $15 \mathrm{sec}$ long, the next five were $10 \mathrm{sec}$ long, and the final six were $5 \mathrm{sec}$ long. The fourth pretraining session was designed to establish leverpressing during the 5 -sec period beginning $5 \mathrm{sec}$ after the termination of the $X$ and $Y$ cues. The reinforcement contingencies were made more stringent in four steps, with four of each kind of trial $(X$ and $Y$ ) given in each step. In the first step, each leverpress during 15-sec $X$ and $Y$ cues was reinforced. In the next step, each leverpress was reinforced during 5-sec presentations of the cue and 10 -sec empty intervals immediately after each cue. Then, leverpresses were reinforced only during the 10-sec empty interval after each cue. Finally, leverpresses were reinforced only during the 5$\mathrm{sec}$ period that began $5 \mathrm{sec}$ after the termination of each cue.

The final pretraining session comprised four presentations of each of the four types of excitatory trials to be used in discrimination training: $\mathrm{A}+, \mathrm{B}+, \mathrm{Y} \rightarrow \mathrm{O}+$, and $\mathrm{B} \rightarrow \mathrm{O}+$. Each leverpress was reinforced during each 5-sec presentation of the A+ and $\mathrm{B}+$ cues and during the 5-sec empty interval that began $5 \mathrm{sec}$ after the termination of each 5 -sec presentation of the $X$ and $Y$ cues.

The rats were then randomly assigned to one of three groups ( $n \mathrm{~s}$ $=8$ ), and discrimination training was begun. Each of the 80 dis- crimination training sessions was $80 \mathrm{~min}$ long and included 10 trials. Each group received six kinds of trials; five were common to rats in all three groups. The first four common trials were identical to those given in the final pretraining sessions: reinforced $\mathrm{A}+$ and $\mathrm{B}+$ target-alone trials, and reinforced $\mathrm{Y} \rightarrow \mathrm{O}+$ and $\mathrm{X} \rightarrow \mathrm{O}+$ feature-alone trials. The fifth type of trial common to all three groups was the primary nonreinforced compound cue, $\mathrm{X} \rightarrow \mathrm{A}-$, in which a $5-\mathrm{sec}$ $X$ presentation was followed, after a 5-sec empty interval, by a 5-sec nonreinforced A presentation. The sixth trial type, which differed in each group, involved nonreinforced presentations of the $Y$ feature. In Group One, those trials comprised a 5 -sec presentation of $\mathrm{Y}$, followed, after a 5-sec empty interval, by a 5-sec presentation of A. In Group Two, those trials comprised a 5-sec presentation of $\mathrm{Y}$, followed, after a 5-sec empty interval, by a 5-sec presentation of B. In Group Con, the 5-sec Y cue was presented without a target.

In the first two discrimination training sessions, there were three presentations of each of the two types of nonreinforced trials, and one of each of the four types of reinforced trials. Unfortunately, responding was not maintained on this schedule, so the rats were given four "refresher" sessions identical to the final pretraining session, and the discrimination contingencies were reintroduced gradually. In the third discrimination training session, there were two of each reinforced trial type and one of each nonreinforced trial type; in the fourth discrimination training session, there were two of each trial type. For Sessions 5-40, the rats received one of each reinforced trial type and three of each nonreinforced trial type in each session. Finally, in Sessions 41-80, the proportion of featurealone trials was halved by, on alternate sessions, replacing the $\mathrm{X} \rightarrow \mathrm{O}+$ reinforced feature-alone trial with an $\mathbf{X} \rightarrow \mathbf{A}-$ nonreinforced compound trial, or by replacing the $\mathrm{Y} \rightarrow \mathrm{O}$ + reinforced feature-alone trial with the group-appropriate nonreinforced $Y$ trial.

After the completion of discrimination training, all rats received four tests of responding to the individual events and compounds, identical to the first four sessions of Experiment 1

Data analysis. The data were analyzed in the same manner as in Experiment 1. In addition, we present the latency to respond on feature-alone trials. This latency was calculated from the beginning of the 5 -sec interval that started $5 \mathrm{sec}$ after the termination of the feature cue. Thus, it corresponds to the latency of responding to the target on feature $\rightarrow$ target serial compound trials, but the beginning of the interval was unmarked by any explicit event.

\section{Results and Discussion}

Relative to the rate of feature negative discrimination learning in Experiment 1, acquisition of negative patterning proceeded slowly, especially with the B target. This observation may be contrasted to that of Rescorla (1991b), who found that separate reinforced presentations of the feature produced more rapid discrimination learning in a similar pigeon autoshaping experiment. However, in Rescorla's experiment, the reinforcer was delivered immediately after the feature cue, rather than during the interval in which the target would normally be presented. Thus, the excitatory and negative modulatory powers of that feature were likely to be confined to different time periods, minimizing response competition.

By the end of discrimination training, the rats in Groups Two and Con responded with slower latencies during the A target on nonreinforced compound trials than on reinforced target-alone trials $[T \mathrm{~s}(8) \leq 1]$; however, the corresponding differences in Group One were not reliable $[T s(8)=9]$. Discrimination performance over the final five sessions of training is shown in Table 1. As in Ex- 
periment 1 , training was halted at numerically poor levels of discrimination performance intentionally, to minimize ceiling effects during subsequent summation tests.

Figure 2 shows, combined over the four test sessions, the response latencies on compound, target-alone, and feature-alone trials. Consider first the performance of Group One (top panel), in which the $X$ and $Y$ features were both trained with the A target. The right bars show responding during the empty interval after $\mathrm{X}, \mathrm{Y}$, and $\mathrm{XY}$ feature-alone trials, when a target would have been delivered in training. The excitatory tendencies controlled by $\mathrm{X}$ and $\mathrm{Y}$ alone in that interval summed: The latency of responding in that 5-sec interval was significantly shorter after the $\mathrm{XY}$ compound than after either $\mathrm{X}$ or $\mathrm{Y}$ alone $[T \mathrm{~s}(8) \leq 5]$.

Although neither of the individual features reliably slowed responding to the subsequent $A$ target $\left[T_{\mathrm{s}}(8) \geq\right.$ 13; see Figure 2, left bars], the XY compound did [ $T(8)$ $=5]$. Furthermore, the compound feature slowed responding more than did either $\mathrm{Y}$ or $\mathrm{X}$ alone $[T \mathrm{~s}(8) \leq 5]$. Thus, the abilities of $X$ and $Y$ to suppress responding to A apparently summed. Finally, as in Experiment 1, X, $\mathrm{Y}$, and the $\mathrm{XY}$ compound did not slow responding to the separately trained $B$ excitor $\left[T_{\mathrm{s}}(8) \geq 12\right.$; see Figure 2, center bars]. That is, there was no transfer of negative occasion setting to a separately reinforced excitor.

The center panel of Figure 2 shows the test performance of Group Two, in which the $X$ feature had been trained with the $A$ target, and the $Y$ feature had been trained with the B target. As in Group One, the excitatory powers of the $Y$ and $X$ features summed: The latency of responding in the appropriate 5-sec test interval was significantly shorter after the $\mathrm{XY}$ compound than after either $\mathrm{X}$ or $\mathrm{Y}$ alone $[T s(8) \leq 6]$.

Substantial transfer of the features' occasion-setting power was evident in Group Two: $X$ reliably slowed responding to the B transfer target $[T(8)=1$; Figure 2, center bars] and $\mathrm{Y}$ significantly slowed responding to the A transfer target $[T(8)=5$; Figure 2, left bars]. Although the $\mathrm{X}$ feature slowed responding to its original target more than responding to its transfer target $[T(8)=0]$, the $Y$ feature did not $[T(8)=24]$. As in Group One, the negative occasion-setting powers of the two features summed: The XY compound slowed responding to both the A target and the $B$ target more than did either feature alone $[T \mathrm{~s}(8) \leq 5.5]$. However, unlike in Experiment 1, the summation effect did not differ in magnitude from that in Group One $[U(8,8)=31.5]$.

The bottom panel of Figure 2 shows the test performance of Group Con, in which the $\mathrm{X}$ feature had been trained in a negative patterning discrimination with the A target, but the $Y$ cue was simply nonreinforced (and thus not trained as an occasion setter). Not surprisingly, the nonreinforced $\mathrm{Y}$ did not add to the excitatory powers of $X[T(8)=14$; right bars]. More important, $Y$ did not slow responding to either the A target (left bars) or the $B$ target (center bars) $[T \mathrm{~s}(8) \geq 14]$, and indeed $Y$ inter-
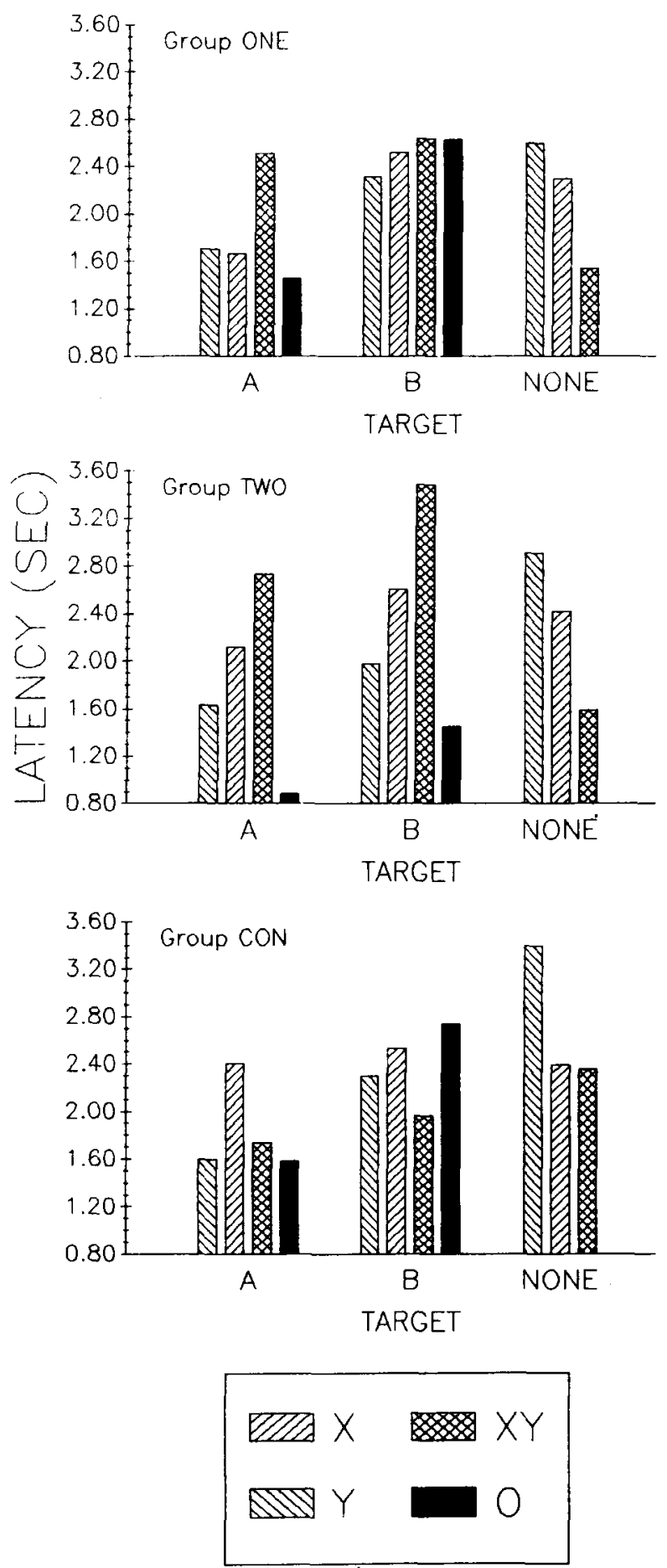

Figure 2. Test performance in Experiment 2. In each panel, the left set of bars shows responding on test trials that included the $A$ (noise/tone) target, the center set of bars shows responding on test trials that included the B (panel light) target, and the right set of bars (labeled NONE) shows responding during the equivalent empty trace interval on feature-alone test trials. Shading of the bars (see legend) signifies whether a target (or empty trace interval) was preceded by the X (door light), Y (clicker), XY (clicker + door light compound), or no $(O)$ feature. 
fered with X's ability to slow responding to A $[T(8)=$ 6]. Furthermore, the difference between responding during the A target after the $X Y$ compound feature and after the $X$ feature, the measure of summation of negative occasion setting, was reliably greater in both Group One $[U(8,6)=5]$ and Group Two $[U(7,6)=11]$ than in Group Con. Thus, the summation of negative occasion setting observed in Groups One and Two was due to the training contingencies of those groups, rather than the mere presence of the two features at the time of testing. Finally, as in Group One, the $\mathrm{X}$ feature failed to significantly slow responding to the separately reinforced $B$ target $[T(8)=23]$.

Comparisons of summation and transfer among the groups are weakened somewhat by the different levels of responding controlled by the transfer $B$ target among the groups: The rats in Group Two showed reliably shorter latencies than did the rats in the other two groups $[U \mathrm{~s}(7,8$ or 6$) \leq 10$ ]. However, it is worth noting that despite their shorter latency to respond to B alone, the rats in Group Two showed reliably longer latencies to respond to that target when it was preceded by the XY compound feature than did the rats in the other two groups $\left[U_{\mathrm{s}}(7,8\right.$ or $6) \leq 101$. Thus, the greater power of the $X Y$ feature to suppress responding to the B target in Group Two could not be attributable to ceiling and other level effects.

In summary, the pattern of transfer and summation in this negative patterning procedure was similar to that observed with feature negative discriminations in Experiment 1 . Transfer was substantial to targets of other negative occasion setters, but did not occur with separately reinforced target stimuli. This pattern of transfer is damaging to a casual configural account of negative patterning learning, which might posit the establishment of inhibition to a cue unique to the nonreinforced compound as the individual elements acquire excitation. Reduced responding to a transfer test compound would be anticipated only to the extent that this unique cue generalizes to the transfer test compound. Moreover, transfer would not be anticipated to depend on the training history of the target: The transfer test compound would differ from the training compound equally regardless of that history.

The negative occasion-setting powers of features summed, both when the features had been trained with the same target and when they had been trained with different targets. This summation of the features' suppressive powers on feature-target trials was especially impressive because it occurred despite the summation of those features' excitatory control over responding on featurealone trials.

\section{GENERAL DISCUSSION}

With both feature negative and negative patterning procedures, negative occasion setting transferred readily (but not perfectly) to cues trained as targets of other occasion setters, but not to cues that were separately and consistently reinforced. Furthermore, after both feature nega- tive and negative patterning training procedures, the features' negative occasion-setting powers summed, both when the features had been trained with the same targets and when they had been trained with different targets. These data extend previously reported data from Pavlovian feature negative procedures (e.g., Holland, 1989), which showed transfer to be similarly dependent on the training history of the test target.

Even under conditions amenable to transfer, however, specific feature-target relations were evidently encoded. Transfer was incomplete in both experiments. With feature negative procedures in Experiment 1, summation of negative occasion setting was better when the occasion setters were trained with the same target than when they were trained with different targets. Any adequate theory of occasion setting must deal with both these specific and general aspects of transfer; unfortunately, none of the current accounts does so in a simple manner (e.g., Holland, 1983, 1992; Pearce, 1987; Rescorla, 1985, 1992).

In Experiment 2, which used negative patterning discrimination training procedures, the summation of negative occasion setting occurred despite the summation of the features' excitatory stimulus control. The latter observation not only makes that demonstration of summation of negative occasion setting especially convincing, it extends previous demonstrations of the independence of the simple conditioning and occasion-setting functions of feature cues (e.g., Holland, 1984, 1989, 1991; Rescorla, 1985).

It is worth comparing our results with those recently described by Rescorla (1991a, Experiment 1). In that autoshaping experiment, pigeons were trained with four separate feature negative discriminations, involving four features and two targets. Then, performance during a separately trained and partially extinguished target was examined after various compounds of two features. Responding to the test target was substantially more suppressed after compounds of features that had been originally trained with a common target than after compounds of features that had been trained with different targets. Although this outcome is reminiscent of our finding in Experiment 1 that summation of negative occasion setting was greater when the two features had been trained with a common target than when they were trained with different targets, two differences in the experiments should be noted.

First, the procedure used to establish Rescorla's (1991a) test target, training, and partial extinction does not generate a target that is influenced by negative occasion setters in our laboratory (e.g., Lamarre \& Holland, 1987). The summation effects we reported here did not extend to separately trained cues (which underwent substantial extinction over the course of the multiple test sessions).

Second, in our experiments, summation was assessed with the targets from original training. Whereas the subjects in Group One had previously experienced the test target after each of the individual elements of the compound test feature, the combination of one of the feature 
elements with the test target was novel for the subjects in Group Two. This greater novelty of the test situation in Group Two might have altered the magnitude of the transfer effect, for example, by disrupting the normal summation of negative occasion setting (artificially reducing the apparent magnitude of summation) or by producing greater disruption of leverpressing (artificially enhancing the summation effect). Although these possibilities do not mitigate the conclusion that specific feature-target relations were encoded (the novelty of the test compound could not be detected unless the original features were encoded), they do suggest caution in interpreting the origin of the reduced summation observed when features trained with different targets were combined in Experiment 1. Recall, however, that Rescorla (1991a) also found better summation with a common target, while avoiding the problem of differential generalization decrement by using a test target that had never been presented with any of the features.

The results of our experiments are relevant to accounts of occasion-setting phenomena based on configuration and generalization of simple excitatory and inhibitory tendencies. For example, Pearce (1987) argued that subjects treat each stimulus event as a unique stimulus configuration. Various summation and transfer effects are the consequence of generalization of excitation and inhibition among those configurations, and between configurations and the stimuli that other theorists would describe as the elements of those compounds. Pearce's account has been quite successful in explaining many (but not all) of the phenomena attributed to modulatory processes by Rescorla $(1985,1992)$ and Holland $(1983,1992)$, without recourse to such processes (e.g., Holland, 1992; Wilson \& Pearce, 1990).

Simulations of the procedures of Experiment 1 with Pearce's (1987) model generated many of the qualitative aspects of those data, including the summation of negative occasion setting in Groups One and Two, and the greater summation in Group One than in Group Two. However, those same simulations yielded substantial summation in Group Con as well, which was not observed. Furthermore, the observation in Experiment 1 of greater transfer to a cue when it was trained as a target of another occasion setter relative to when it was separately trained was simulated only for one of the test compounds, and only within a limited set of parameters (Wilson \& Pearce, 1990 , noted a similar problem). Finally, simulations of the procedures of Experiment 2 failed to yield simultaneous summation of negative occasion setting and excitatory tendencies with any parameter values.

Network theories of configural learning may fare better with these data. Consider, for example, Kehoe's (1988) account for the solution of negative patterning (XA-/A+l $\mathrm{X}+$ ) discriminations within a multilayer network model. In that model, responding to a CS is mediated by connections between an input unit corresponding to that $\mathrm{CS}$ and various "hidden units," and by connections between those hidden units and an output unit. Activation of a unit depends on the (probabilistic) sum of its inputs, as well as the value of a characteristic threshold for that unit.

In an $\mathrm{XA}-/ \mathrm{A}+/ \mathrm{X}+$ discrimination, excitatory connections (connection weights of positive value) are established between the A and X CS-input units and each of two hidden units, which converge on a single output unit. As a further consequence of the patterning contingencies, the connection weight between one of the hidden units and the output unit is driven negative and that between the other hidden unit and the output unit is driven positive (we will refer to these two hidden units as the inhibitory and excitatory hidden units, respectively). Solution of the discrimination entails a high activation threshold for the inhibitory hidden unit, such that $\mathrm{X}$ and $\mathrm{A}$ individually activate only the excitatory hidden unit, whereas the joint presentation of $\mathrm{X}$ and $\mathrm{A}$ activates both hidden units. Because the activity of the output unit depends on the sum of its inputs (both inhibitory and excitatory), responding will be less likely in the presence of the XA compound than during the individual elements.

If this model is applied to the experimental designs described here by assuming that all CS-input units share the same two hidden units, it accurately predicts a number of our observations. First, because the separately trained cue was never presented nonreinforced or in compound with another cue, it would have negligible connections with the inhibitory hidden unit. Thus, no transfer of an occasion setter's power to such a target would be anticipated. Indeed, the model makes the further prediction that transfer would occur only to targets whose training led to substantial connections with the inhibitory unit-in other words, targets with a substantial inhibitory component. Rescorla (1987) made a similar claim. Second, because the cues involved in the negative patterning discriminations are all connected to common hidden units, substantial transfer to similarly trained targets would be anticipated. (Note that our observations of some specificity of coding even among like-treated targets suggest that the hidden units are not shared completely.)

Third, if a number of conditions are met, the model can accommodate the simultaneous summation of the features' excitatory and negative occasion-setting powers, which we observed in Experiment 2. If the sum of X's and A's connections with the inhibitory hidden unit were barely above threshold, and the sum of those cues' connections with the excitatory hidden unit were substantially above threshold, then (because of the probabilistic nature of summation in the model) addition of a feature from another negative patterning discrimination to the XA compound could yield a greater increase in the activity of the inhibitory hidden unit than in the excitatory hidden unit. Thus, summation of negative occasion setting is consistent with the model. If, at the same time, the sum of the $\mathrm{X}$ and $\mathrm{Y}$ features' connections with the inhibitory unit was 
subthreshold, combining the $\mathrm{X}$ and $\mathrm{Y}$ features in the $\mathrm{ab}$ sence of a target would yield summation of those features' excitation.

In order to simultaneously satisfy both of the aforementioned conditions, the weight of the connection between the target and the inhibitory hidden unit must be substantial-indeed, greater than that of the feature's connection with that unit. Given such substantial inhibitory contributions of the targets, the model makes the further prediction that summation of occasion setting would be observed with a compound of two targets and one feature. That is, responding would be less during $X \rightarrow A B$ than during $\mathrm{X} \rightarrow \mathrm{A}$. Regrettably, we did not test compounds of target cues in this experiment.

The correspondences just described are likely to be highly dependent on the values of the various thresholds and weights within the model, and hence many training parameters. Note that Maki and Abunawass (1991) presented a very similar model of matching-to-sample performance, which, consistent with the data of Maki and Leuin (1972, described earlier), predicts that features should compete with each other rather than sum. We have conducted only crude simulations of our procedures and cannot vouch for whether the assumptions we made yield many other basic properties of conditioning and occasion setting in our preparation. Nor have we considered how to deal with our consistent observation that the outcomes of various manipulations depend dramatically on the temporal arrangement of feature and target in training. But these issues are clearly worthy of further empirical and theoretical exploration. Of critical importance is whether the different results that we have attributed to differences between simple conditioning and occasion setting can be reconciled to a single set of excitatory and inhibitory processes or whether it is necessary to postulate a modulatory process distinct from simple conditioning, as Holland $(1983,1992)$ and Rescorla $(1985,1992)$ have done.

In this regard, it is worth noting that some network models of conditioning already incorporate distinctions between elicitation and modulation. For example, both Grossberg's extensive model (e.g., Grossberg \& Schmajuk, 1989; Schmajuk \& DiCarlo, 1991) and Wagner and Brandon's (1989) model involve modulation of the output of specific sensory representations by the activity of more general drive representations. Although the modulatory processes in those two models differ substantially, and both differ from those proposed by Holland (1983, 1992) and Rescorla $(1985,1992)$, the multilayer structures they envision are compatible with many of the claims made by the latter investigators about the nature of representation of events in occasion setting.
Holland. P. C. (1983). Occasion-setting in Pavlovian feature positive discriminations. In M. L. Commons, R. J. Herrnstein, \& A. R. Wagner (Eds.), Quantitative analyses of behavior: Discrimination processes (Vol. 4, pp. 183-206). New York: Ballinger.

Holland, P. C. (1984). Differential effects of reinforcement of an inhibitory feature after serial and simultaneous feature negative discrim ination training. Joumal of Experimental Psychology: Animal Behavior Processes, 10, 461-475

Holland, P. C. (1985). The nature of conditioned inhibition in serial and simultaneous feature negative discriminations. In R. R. Miller \& N. E. Spear (Eds.). Information processing in animals: Conditioned inhibition (pp. 267-297). Hillsdale, NJ: Erlbaum.

Holland, P. C. (1989). Transfer of negative occasion setting and conditioned inhibition across conditioned and unconditioned stimuli. Journal of Experimental Psychology: Animal Behavior Processes, 15. 311-328.

Holland, P. C. (1991). Acquisition and transfer of occasion setting in operant feature positive and feature negative discriminations. Learning \& Motivation, 22, 366-387.

Holland, P. C. (1992). Occasion setting in Pavlovian conditioning. In D. Medin (Ed.), The psychology of learning and motivation (Vol. 28, pp.69-125). San Diego: Academic Press.

KEHOE, E. J. (1988). A layered network model of associative learning: learning to learn and configuration. Psychological Bulletin, 95. $411-422$.

LamarRe, J., \& Holland, P. C. (1987). Acquisition and transfer of serial feature negative discriminations. Learning \& Motivation, 18. 319-342

MAKI, W. S., \& AbUNAwass, A. M. (1991). A connectionist approach to conditional discriminations: Learning, short-term memory, and attention. In M. A. Commons, S. Grossberg, \& J. E. R. Staddon (Eds.), Neural network models of conditioning and action (pp. 241278). Hillsdale, NJ: Erlbaum.

MAKI, W. S., \& LEUIN, T. C. (1972). Information processing in pigeons. Science, 176, 535-536

Pearce, J. M. (1987). A model for stimulus generalization in Pavlovian conditioning. Psychological Review, 94, 61-75.

RESCORLA, R. A. (1985). Conditioned inhibition and facilitation. In R. R. Miller \& N. E. Spear (Eds.), Information processing in animals: Conditioned inhibition (pp. 299-326). Hillsdale, NJ: Erlbaum.

RESCORLA, R. A. (1987). Facilitation and inhibition. Journal of Experimental Psychology: Animal Behavior Processes, 13, 250-259.

REsCorla, R. A. (1991a). Combinations of modulators trained with the same and different target stimuli. Animal Learning \& Behavior, 19, 355-360.

RESCORLA, R. A. (1991b). Separate reinforcement can enhance the effectiveness of modulators. Journal of Experimental Psychology: Animal Behavior Processes, 17, 259-269.

ResCorla, R. A. (1992). Hierarchical associative relations in Pavlovian conditioning and instrumental training. Current Directions in Psychological Science, 1, 66-70.

Schmajuk, N. A., \& DiCARLo, J. J. (1991). A neural network approach to hippocampal function in classical conditioning. Behavioral Neuroscience, 105, 82-110.

WaGNER, A. R., \& BRANDON, S. E. (1989). Evolution of a structured connectionist model of Pavlovian conditioning (AESOP). In S. B. Klein \& R. R. Mower (Eds.), Contemporary learning theories: Pavlovian conditioning and the status of traditional learning theory (pp. 149189). Hillsdale, NJ: Eribaum.

Wilson, P. N., \& PEARCE, J. M. (1990). Selective transfer of responding in conditional discriminations. Quarterly Journal of Experimental Psychology, 42B, 41-58.

\section{REFERENCES}

Grossberg, S., \& Schmajuk, N. A. (1989). Neural dynamics of adaptive timing and temporal discrimination during associative learning. Neural Networks, 2, 79-102.
(Manuscript received June 1, 1992; revision accepted for publication October 9, 1992.) 